\title{
Decentralized Multi-Agent System Applied to the Decision Making Process of the Microgrid Restoration Procedure towards Sustainability
}

\author{
Ebrahim Rokrok, Miadreza Shafie-khah, \\ Gerardo Osório \\ C-MAST/UBI, Covilha, Portugal \\ ebrahim.rokrok@gmail.com \\ miadreza@ubi.pt \\ osorio.silva@ubi.pt
}

\author{
João Carvalho \\ FEUP, Porto, \\ Portugal \\ pedrojo.carvalho@gmail.com
}

\author{
João P. S. Catalão \\ INESC TEC and FEUP, Porto, \\ C-MAST/UBI, Covilha, and \\ INESC-ID/IST-UL, Lisbon, \\ Portugal \\ catalao@fe.up.pt
}

\begin{abstract}
A significant procedure to ensure the consumer supply is Power System Restoration (PSR). Due to the increase of the number of distributed generators in the grid, it is possible to shift from the conventional PSR to a new strategy involving the use of distributed energy resources (DER). In this paper, a decentralized multiagent system (MAS) is proposed to cope with the restoration procedure in a microgrid (MG). Each agent is assigned to a specific consumer or microsource (MS), communicating with other agents at every stage of the restoration procedure so that a common decision is reached. The 0/1 knapsack problem is the problem that every agent solves to determine the best load connection sequence during the restoration of the MG. Two different case studies are used to test the MAS on a dynamically modeled benchmark MG: a total blackout and a partial blackout. Regarding the partial blackout case, demand response emergency programs are considered to manage the loads in the MG. The MAS is developed in Matlab/Simulink environment and by performing the corresponding dynamic simulations it is possible to validate this system towards sustainability.
\end{abstract}

\section{Introduction}

\subsection{Aims and Motivation}

The limitation of the operational margins of power systems led to an increase on the risk of power outages and blackouts. On this subject, one of the most important problems to be analyzed is the power system restoration (PSR).
Considering the conventional procedure, this process begins with the energization of the transmission system by starting the black-start units/power plants. Possible examples of black-start units are hydroelectric units, gas and fuel turbine units. A black-start feature can also be the incoming power from the remaining interconnected systems in the vicinities [1]. Using this procedure, the transmission system will be energized and then the consumers near the power plants will be supplied first, meaning that the distribution system's consumers must wait longer to be supplied.

Over the years, a restructuring of the power systems has been verified, regarding the integration of dispersed generation units (renewable and nonrenewable) in the distribution systems, which led to the identification of the relatively new concept called microgrid (MG). As a result, the power flow in a $\mathrm{MG}$ is now bi-directional and the islanded operation is also enabled when necessary [2].

The bi-directional power flow feature can be very useful regarding PSR [3]. Furthermore, it is possible to supply the consumers at the distribution system much faster than the conventional way, since the MG can start supplying low voltage consumers at the same time that the transmission system is being restored. Concluding, by combining the distributed energy resources (DER) with the flexibility of the consumptions in the $\mathrm{MG}$, the restoration process can be improved significantly.

\subsection{Literature Review}

Several studies have been addressed with the purpose of using the advantages of the MG capabilities regarding PSR. Some of these studies regard multiagent system (MAS), integration of electric vehicles (EV) using stochastic methods, considering spanning tree search and also using complex network theory [1]. 
In [4] the power restoration of a smart grid was performed based on a MAS. Ref. [5] proposes a multiagent scheme to tackle the distribution power system restoration, which compares its results with the results obtained by using a mathematical programming strategy. The conclusions show that the quality of the MAS solution is very similar to the other solution and only uses local information, which is an advantage.

The PSR problem is tackled in a renewable-based MG in [6]. Here the MG operates in island mode as a result of an unscheduled disconnection from the medium voltage grid. The uncertainty of the output of renewable sources and the time that the MG is working on island mode are the main challenges. Two stochastic based methods are proposed to address the scheduling problem. In [7], a stochastic model is proposed where the decision making process of the restoration procedure is supported by a stochastic model, according to a pre-hurricane situation. In the same reference it is possible to find two different strategies: one for complete restoration and one for partial restoration.

Ref. [8] presents a stochastic mixed integer linear program that is able to assess the potential of the blackstart capabilities of microgrids. Spanning tree search methods are also used to tackle the restoration procedure. One example is given in [9] where a graphtheoretic strategy is presented considering the integration of microgrids in the distribution system. In this reference, its main purpose is maximizing the load supply and at the same time minimizing the number of operations of the switching devices. Black-start capabilities of microgrids are assessed in [10], considering a power system where the existent microgrids are sectionalized. The microgrids with enough reserve will be responsible for supplying the unsupplied critical loads by applying a spanning tree search algorithm.

The MG restoration procedure and its feasibility are assessed in [3] by performing dynamic simulations in a modeled MG. In the proposed sequence of actions, the microgrid central controller (MGCC) is responsible for establishing the most adequate sequence of actions to enable a successful procedure, including the black-start of some units, the feeder energization, and finally the reconnection of loads and remaining generation units. The MGCC receives the local information of the local controllers (LC), in which the LC and microsource (MS) controllers are included, regarding the production and consumptions values.

Several other dynamic studies that assume centralized approaches were also performed in the literature. In [11], the restoration of a distribution system that is embedded in a multi-microgrid environment is investigated.
In this case, the most adequate sequence of actions is a responsibility of the autonomous management controller (CAMC). Finally, in [12] and [13], the restoration procedure is applied to real microgrids, which are in the Illinois Institute of Technology and Launceston, Tasmania, respectively.

The centralized control schemes in the restoration procedure have the main disadvantage of when one or more communication links between the agents are lost, the entire restoration procedure may be compromised [14].

Single-point failure is also an issue and the process depends exclusively on the MGCC. These drawbacks can be avoided by introducing the decentralized control scheme. This type of control enables a high level of autonomy of the different MS and loads since each one of them will be associated to a specific agent, which in turn is able to communicate with their neighbors in order to exchange their local information [15]. By using a decentralized control scheme, the MGCC is not required [16].

Differently from what happens in the centralized control scheme, when a communication link is lost, the entire system is still able to work since the agents are all connected between each other through other links. However, the deployment of the local controllers and control schemes requires the existence of high coordination standards, which makes this scheme harder to implement [14], [17].

\subsection{Contributions and Paper Organization}

The novel contributions are threefold:

- A decentralized MAS scheme applied to MG restoration scheme is presented in this paper to separate the restoration task from the MGCC. Therefore, the restoration process is aligned with the goal of task distribution in the MG, which in turn reduces the need for a powerful MGCC to process huge amount of data. In the proposed MAS scheme, it is considered that every single agent is assigned to a specific load or generation unit and these will be communicating with each other to collect the global data of the MG, especially regarding the production and load values.

- After the information sharing process, in order to choose the optimum amount of loads with higher priority, a strategy based on solving a same $0 / 1$ knapsack problem considering a table-based dynamic programming algorithm for each load agent is proposed. Therefore, the best sequence of load connection during the MG restoration process is ensured. 
- The simulations regarding the proposed model are carried out in two case studies: a total blackout where every load and MS is disconnected, and a partial blackout as a result of a fault in the MG when operating in island mode.

The organization of this paper is provided as follows. Section II provides the DER's and MG modeling. In Section III the proposed MAS is described as well as the main assumptions taken in consideration during the restoration sequence. In section IV the results are shown and discussed. Finally, section $\mathrm{V}$ presents the main conclusions taken from this work.

\section{Microgrid Restoration Dynamic Model}

A MG can be seen as a small-scale power system. So, when needed the MG can work in island mode, completely independent from the upstream grid. This is a particular useful feature since it allows to reduce the number of consumers that are not supplied in case of a fault. A typical MG structure can be found in Figure 1.

Probably the most important aspect of the microgrid's control strategy regards its individual controllers. The proper operation of the MG is ensured by these individual controllers, when the $\mathrm{MG}$ is working on island mode. The MGCC enables a centralized control over the MG. The technical and economic constraints of a $\mathrm{MG}$ are managed by this device.

Furthermore, it is located at the low voltage bus of the substation and is responsible for sending set-points to the remaining controllers, the $\mathrm{LC}$ and the $\mathrm{MC}$ devices, with the purpose of maintaining the balance between production and consumption while in island mode.

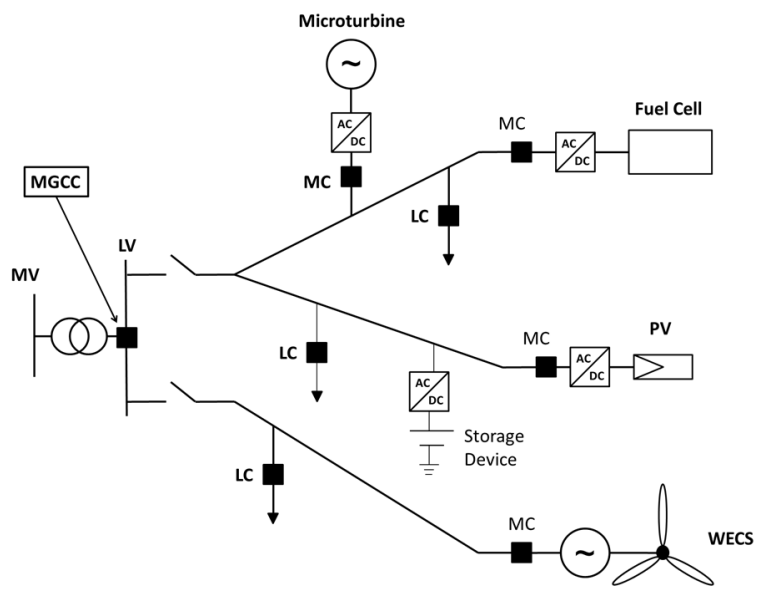

Figure 1. Typical MG structure.
The LC can be found at the consumption points and ensures a local load control with load shedding schemes, in emergency situations. The MC enables the control of the MS's by changing their active and reactive power output injected into the MG [2].

Considering the conventional PSR, a pre-defined restoration plan is already created and is applicated by human operators. So, the operators' experience will determine the time needed for the restoration procedure completion. However, in a MG the available controllers will be in charge of the restoration procedure. By using online information it is possible to reduce the time needed for restoration purposes [3].

The modeled low voltage benchmark MG considered for the simulations in this work can be found in [18]. The MG needs to ensure the power balance during transient periods. This is achieved by using a storage unit, which in this work is considered as a battery installed at the low voltage bus of the substation. Another option to ensure this balance would be to use flywheels; however, in this work this was not considered. The battery was modeled by using a battery model available at the Matlab/Simulink libraries and was connected to a linear $\mathrm{DC} / \mathrm{AC}$ converter. Then, the system is connected to a $\mathrm{V} / \mathrm{f}$ controlled inverter. Regarding the PV generators, these were modeled according to [19] and it was considered that the system is working at its maximum power point through the entire simulations, for pre-specified temperature and irradiance conditions. The model of the wind generator was considered to be an induction machine directly connected to the MG. The wind turbine used in this model is available at the Matlab/Simulink libraries and its equations are available in [20]. The microturbine was considered to be a single-shaft microturbine (SSMT), which possesses a battery at its DC link to provide a fast response during transient periods of the simulations.

This battery is modeled just like the microgrid's main storage unit. The GAST model was used for the turbine model, which can be found in [21], and the generator was considered to be a permanent magnet synchronous generator (PMSG), which can be found in the Matlab/Simulink libraries. Regarding the fuel cell, this MS is assumed to be a solid oxide fuel cell (SOFC) and the model used can be found in [21]. The MSs need to be controlled to adjust the power injected by the units in the MG, which means that the inverters that control these MSs also need to be modeled. The inverters can be modeled as a PQ controlled inverter or a V/f controlled inverter (also known as VSI mode). Regarding the first one, it injects pre-defined values for the injected active and reactive power, while the second one acts like a synchronous generator since it is able to control the voltage and frequency in the $\mathrm{MG}$ using the droop equations presented below: 


$$
\begin{aligned}
& \omega=\omega_{0}-k_{P} \times P \\
& V=V_{0}-k_{Q} \times Q
\end{aligned}
$$

where $\omega_{0}$ regards the reference value of the angular velocity, $V_{0}$ corresponds to the reference value of the voltage magnitude, $P$ and $Q$ represent the active and reactive power output, respectively.

Both $k_{P}$ and $k_{Q}$ constants represent the droop coefficients used for the active and reactive power control, respectively. It is possible to find the control diagrams of both control modes in Figures 2 and 3, respectively.

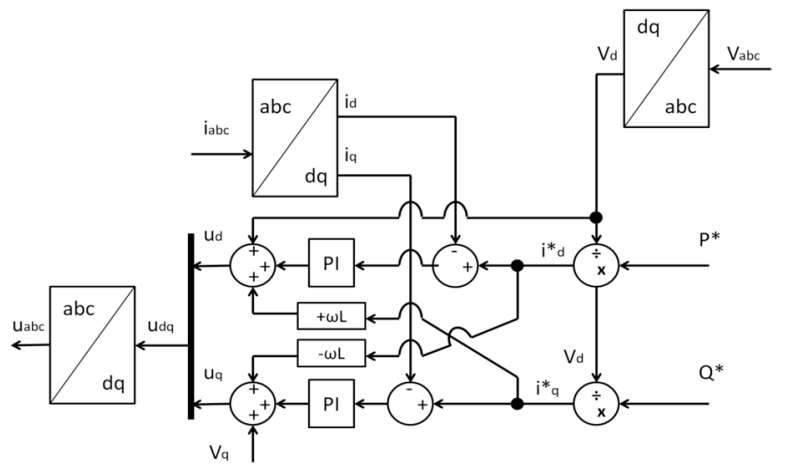

Figure 2. $P Q$ control diagram.

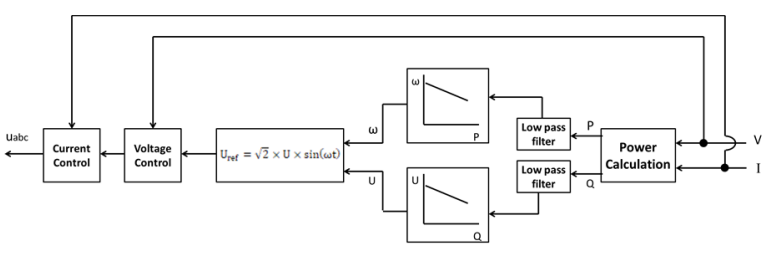

Figure 3. V/f control diagram.

\section{Proposed Decentralized MAS Scheme}

A MAS is characterized by including different agents. According to [22], an agent is a software/hardware system that is able to react to changes in the environment. Furthermore, an agent should be able to adapt itself and observe a part of the current environment.

Considering a $\mathrm{MG}$, the environment that the agents must be aware of will be the power grid, which can be observable by the agents through the use of sensor measurements.

Furthermore, an agent can also change this environment by reconfiguring the network through the opening/closing of a circuit breaker.

According to [23], [22] and [24], an agent must possess three key features in a MG environment:
- Reactivity: the ability to be aware and react to changes in the environment is very important and these changes include, for example, generation output variation, load value variation, and network reconfiguration.

- Pro-activeness: the agents must be able to adapt when a connection is lost, for example, and try to search for another link to other agents that can ensure the same information sharing objectives;

- Social ability: the agents should communicate with the remaining ones to collect the required information. However, it is required that the communication between agents is goal oriented, which requires the cooperation between agents.

\subsection{MAS characterization}

Regarding the restoration procedure of the modeled $\mathrm{MG}$, it is possible to find the structure of the MAS in Figure 4. It can be seen that both the load controllers (LC's) and microcontrollers (MC's) are assigned to each agent and will possess different local information. Considering the LC agent, the load value will be the most important information; however, regarding the $\mathrm{MC}$ agent the most important information will be the available production. In the $\mathrm{V} / \mathrm{f}$ controlled SSMT, the available information will regard its reserve and in the remaining MS its available generation will correspond to its maximum output power.

In [25], the time required for the communication between agents is assessed using a consensus algorithm, which concludes that this time is less than 0.01 seconds. This means that this time is very small when compared to the time intervals between the connection of loads and MS in this MG in such way that it can be neglected. The proposed MAS is assumed to follow the abovementioned consensus algorithm in terms of communication times.

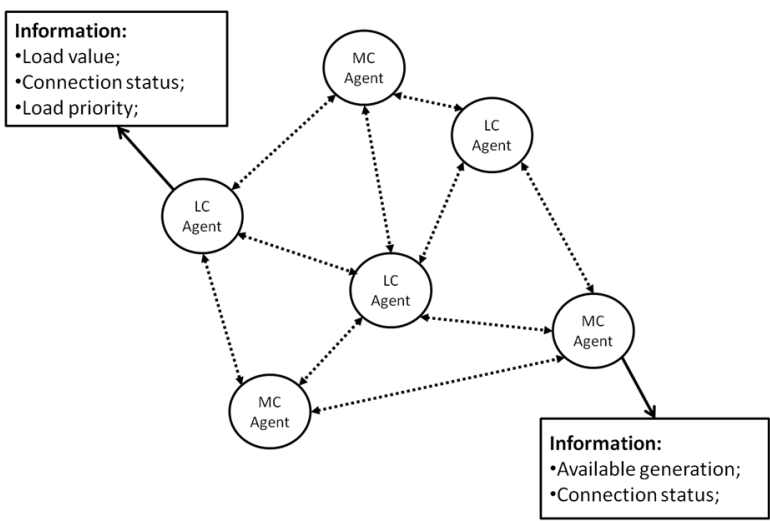

Figure 4. Multi-agent system structure. 


\subsection{Knapsack Problem Formulation}

Regarding the knapsack problem, this consists on an optimization problem that can be applied to the everyday decision making processes [26], [27]. If $N$ items are considered, the problem's objective is to determine how to lay these items in a knapsack.

This knapsack has a maximum capacity $W$, and each item possesses a pre-defined weight $\left(w_{i}\right)$ and value $\left(v_{i}\right)$. So, the objective function will be to maximize the total value placed in the knapsack knowing that the sum of the weights must be less than or equal to $W$.

The adaptation of this problem to the $\mathrm{MG}$ restoration problem can be easily performed considering that $v_{i j}$ represents the priority of the load $j$ of the consumer $i, x_{i j}$ represents a binary variable that has a value of 1 when the corresponding load is connected, $L$ corresponds to the sum of weights and, finally, $w_{i j}$ represents the load value (active power).

The problem is formulated as follows:

objective

function:

$$
\max f(x)=\sum_{i \in N} \sum_{j \in L} v_{i j} \cdot x_{i j}
$$

subject to:

$$
\sum_{i \in N} \sum_{j \in L} w_{i j} \cdot x_{i j}<W
$$

The presented strategy is based on solving the knapsack problem every time a different load is connected to the MG. All agents will solve this problem and reach a common solution. The structure of the agents is presented in Figure 5.

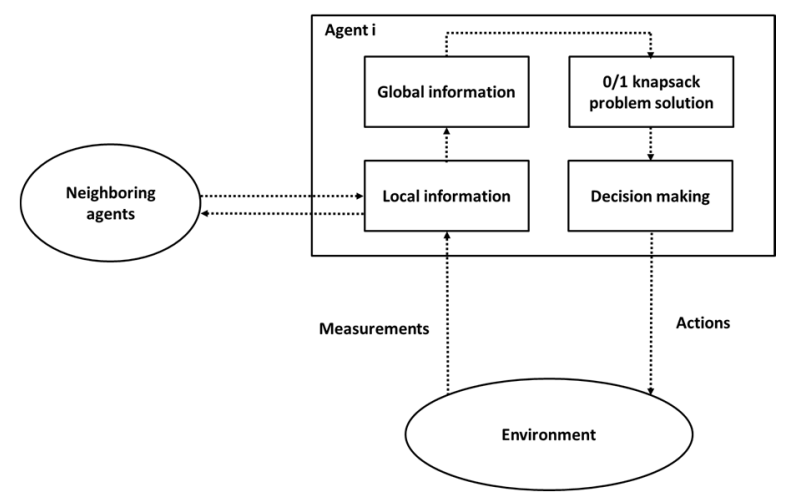

Figure 5. Structure of an agent for MG restoration.

Considering that the SSMT and storage unit were already connected together and synchronized, the proposed algorithm is the following:

1. Update $W$ with the available active power production in the first stage with the SSMT reserve value;
2. Solve the $0 / 1$ knapsack problem, thus determining the most suitable loads to connect, based on their priorities;

3. Discard the loads that were connected previously from the input section of the algorithm, and only consider the loads that are still not connected;

4. The next step is to connect the MS with the highest available capacity; if more than one exists having the same capacity, choose randomly between them;

5. Update $W$. This variable will always correspond to the SSMT reserve since the control of this MS follows a V/f control strategy. Meaning that, after connecting a MS the SSMT will always continuously decrease its output to maintain the frequency at exactly $50 \mathrm{~Hz}$, thus increasing the available reserve. When a load is connected, the opposite situation occurs;

6. Redo step 2 to 5 until no more MS are available to be connect and the SSMT's active power reserve is not enough to connect more loads to the MG.

The storage unit is not considered as a MS with reserve since its main purpose is to only act during transient periods, meaning that its output will return to zero after connection/disconnection of loads or MS.

\subsection{Dynamic Programming}

Solving the 0/1 knapsack problem involves using the table-based dynamic programming algorithm. The Bellman's equation is the base of this algorithm [26]:

$$
f_{j}(i)=\max \left(p_{j}+f_{j-1}\left(i-w_{j}\right), f_{j-1}(i)\right)
$$

where, taking into consideration the knapsack problem, $f_{j}(i)$ regards the maximum value that resulted from the previously presented objective function of the knapsack problem. It is necessary that every coefficient is an integer and the items need to be sorted in an ascending order according the items' weights.

In [26], more information and examples can be found regarding the table-based dynamic algorithm.

\subsection{Load Priority}

To define the load priority, it is necessary to describe every existing type of residential loads, of the MG. The most common types of residential loads, divided by groups, can be found in [28]. See Table 1. 
Here, each of the load groups possess a maximum off time which regards the maximum time that these loads can be turned off without causing any kind of discomfort to the consumers. To be able to minimize the consumers' satisfaction, the main assumption was that the loads with lower maximum off time correspond to higher priorities. The contrary situation occurs with the loads with higher maximum off times. These assumptions can be found in [29] and were adopted in this paper. For clarification purposes, a load $i . j$ will regard the group of loads $\mathrm{j}$ of the consumer $i$. The distribution of load for each consumer is given in Table 2, while the amount of load for every group is provided in Table 3.

In the initial stages of the restoration process, the SSMT is responsible for supplying a pre-defined local load. This local load will correspond to the loads 5.2, 5.3, 5.4 and 5.5, since these have the highest priorities in consumer 5, which means that these are not considered in the knapsack problem since they were already connected, and load 5.1 cannot be supplied since this MS does not have enough capacity $(30 \mathrm{~kW})$.

Table 1. Individual domestic load groups characterization.

\begin{tabular}{|l|l|l|l|}
\hline Group & Description & $\begin{array}{l}\text { Maximum } \\
\text { off time }\end{array}$ & Priority \\
\hline 1 & $\begin{array}{l}\text { Electric space and } \\
\text { water heaters, refrigerators, } \\
\text { freezers }\end{array}$ & 5 minutes & 1 \\
\hline 2 & $\begin{array}{l}\text { Washing machines, } \\
\text { tumble dryers }\end{array}$ & 3.5 minutes & 2 \\
\hline 3 & Cooking appliances & 2 minutes & 3 \\
\hline 4 & In line heaters & 15 seconds & 4 \\
\hline 5 & Lighting loads & 4 seconds & 5 \\
\hline
\end{tabular}

Table 2. Distribution of load for each consumer.

\begin{tabular}{|c|c|c|c|c|c|}
\hline Consumer & Group & Group & Group & Group & Group \\
& $\mathbf{2}$ & $\mathbf{3}$ & $\mathbf{5}$ \\
\hline 1 & $60 \%$ & $11 \%$ & $12 \%$ & $9 \%$ & $8 \%$ \\
\hline 2 & $59 \%$ & $12 \%$ & $11 \%$ & $9 \%$ & $9 \%$ \\
\hline 3 & $62 \%$ & $10 \%$ & $9 \%$ & $10 \%$ & $9 \%$ \\
\hline 4 & $63 \%$ & $9 \%$ & $10 \%$ & $8 \%$ & $10 \%$ \\
\hline 5 & $65 \%$ & $9 \%$ & $8 \%$ & $8 \%$ & $10 \%$ \\
\hline
\end{tabular}

Table 3. Amount of load for every group.

\begin{tabular}{|c|c|c|c|c|c|c|}
\hline Consumer & $\begin{array}{c}\text { Total } \\
(\mathbf{k W})\end{array}$ & $\begin{array}{c}\text { Group } \\
\mathbf{1} \\
(\mathbf{k W})\end{array}$ & $\begin{array}{c}\text { Group } \\
\mathbf{2} \\
(\mathbf{k W})\end{array}$ & $\begin{array}{c}\text { Group } \\
\mathbf{3} \\
(\mathbf{k W})\end{array}$ & $\begin{array}{c}\text { Group } \\
\mathbf{4} \\
(\mathbf{k W})\end{array}$ & $\begin{array}{c}\text { Group } \\
\mathbf{5} \\
(\mathbf{k W})\end{array}$ \\
\hline 1 & 4.845 & 2.907 & 0.532 & 0.581 & 0.436 & 0.387 \\
\hline 2 & 19.55 & 11.534 & 2.346 & 2.150 & 1.759 & 1.759 \\
\hline 3 & 21.25 & 13.175 & 2.125 & 1.912 & 2.125 & 1.912 \\
\hline 4 & 4.845 & 3.052 & 0.436 & 0.484 & 0.387 & 0.484 \\
\hline 5 & 48.45 & 31.492 & 4.360 & 3.876 & 3.876 & 4.845 \\
\hline
\end{tabular}

The storage unit is assumed to be supplying the lighting loads, since these have the lowest maximum time off, which means that they have the highest priority.

These loads are relatively small, so the storage is able to stabilize the frequency in its island, only a bit below $50 \mathrm{~Hz}$. The SSMT has secondary frequency control, so the frequency will be restored to $50 \mathrm{~Hz}$.

\section{Results and Discussion}

The dynamic simulations are carried out in this section to assess the feasibility of the proposed MAS, applied to MG restoration. It is possible to find the low voltage benchmark MG used in this work, implemented in Matlab/Simulink environment, in Figure 6, and its electrical features are available in [18].

\subsection{Total Blackout}

The main assumption in this case study is that the MG is in complete blackout status, which means that all the MS and loads are disconnected from the MG.

Namely, the first steps of the restoration procedure of the MG are: the storage unit needs to energize the feeders of the grid and supply the critical loads, whose priority was defined previously, and the SSMT must be black-started and supply a pre-defined local load, which results in the creation of two islands.

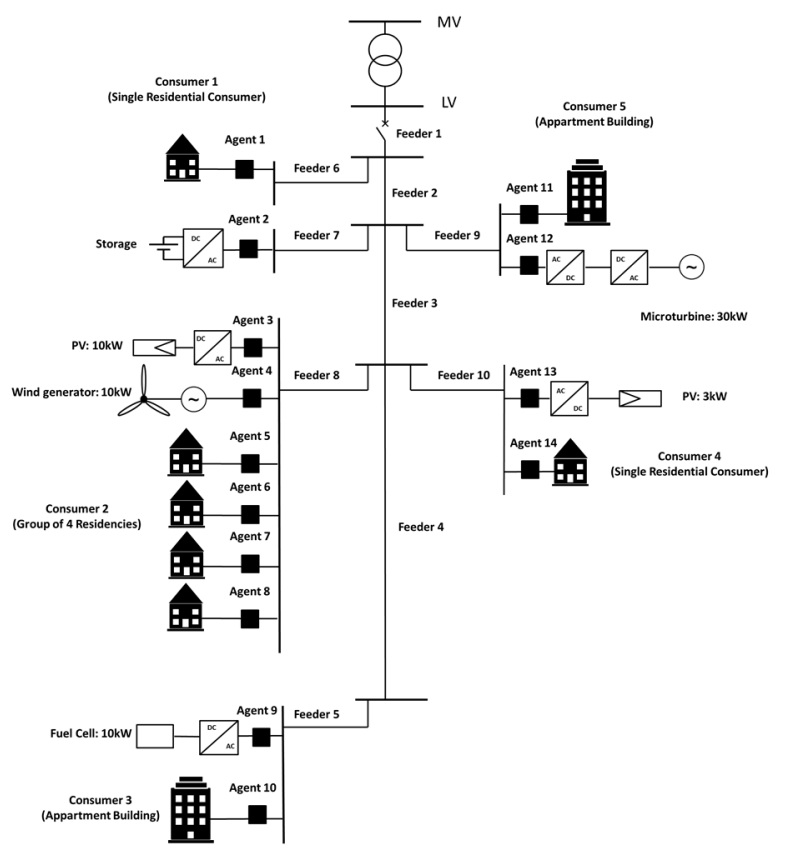

Figure 6. Benchmark low voltage MG. 
The next step would be to synchronize both islands. The MGCC is assumed to be the responsible unit for these initial steps. Regarding the synchronization process, it is necessary to assess an appropriate set of conditions to be able to choose the most suitable moment to connect both islands together.

The synchronization conditions consist in verifying the voltage magnitude and phase differences. By verifying the synchronization conditions, the synchronization procedure will not cause any major transient in the MG, whether in terms of voltage or frequency. The synchronization results can be found in Figure 7.

Prior 2.5 seconds, both the storage unit and SSMT are not connected from the MG. At exactly 2.5 seconds, the MGCC will send a signal to the circuit breakers of these two microsources so that the storage unit can supply the loads with the highest priority and the SSMT can supply a pre-defined local load. This means that two separate islands will be created.

Due to the secondary control of the SSMT, the frequency in its island will be corrected. Since the same frequency control is not applicable to the storage unit, its island will maintain a frequency of approximately $49.85 \mathrm{~Hz}$. The frequency in the SSMT's island will eventually be completely restored, which occurs at 4.5 seconds, and at this instant the MGCC sends a signal to verify the synchronization conditions. Finally, at 5 seconds these conditions are satisfied and so the MGCC sends a signal to the circuit breaker to close and, therefore, connect the two islands. The proposed MAS is used after this synchronization procedure to determine the best load connection sequence. The knapsack problem will be solved at every stage of the restoration process and then the selected loads will be connected.

In Figure 8 the load connection results are presented, where it can be observed the effect of the losses, specifically at 14 seconds and 24 seconds, since at these instants the SSMT's active power output is over its capacity $(30 \mathrm{~kW})$.

Considering the first stage of the load connection sequence, the SSMT's reserve will be $6 \mathrm{~kW}$ and the algorithm selects $6 \mathrm{~kW}$ of load to connect; however, the SSMT will produce over $6 \mathrm{~kW}$, thus exceeding its capacity.

Since the SSMT is able to produce more than its maximum capacity for short periods of time, this effect is not of utmost importance in these simulations.

The remaining results regarding the MG's voltage and active power output of the MS are presented in Figure 9. By checking this figure, it can be seen that the MS that causes the biggest voltage and frequency transients when it is connected to the MG is the wind generator, as it can be seen at 39 seconds.

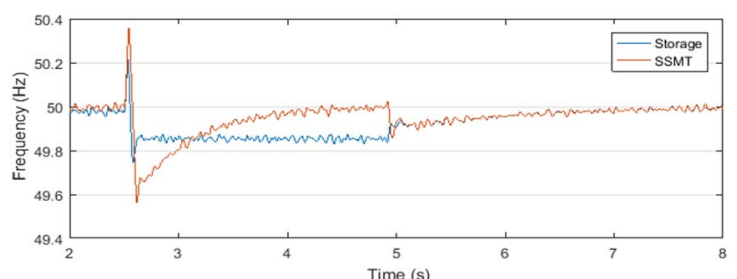

(a)

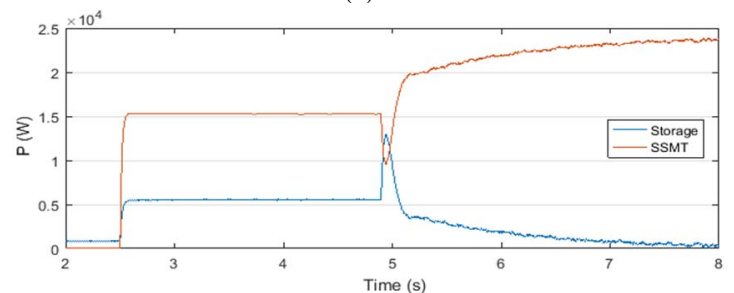

(b)

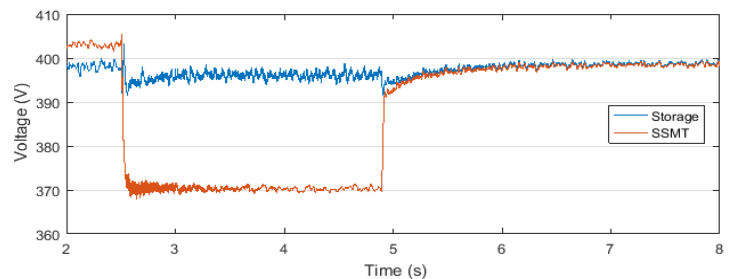

(c)

Figure 7. Synchronization time span: a) frequency, b) storage and SSMT active power output, c) storage and SSMT voltages.

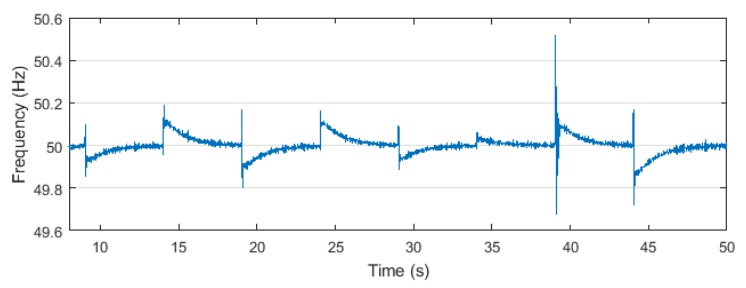

(a)

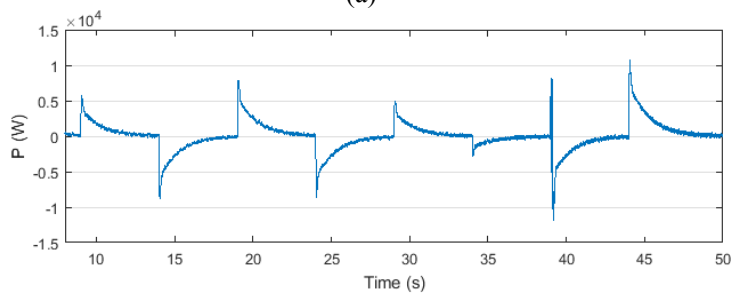

(b)

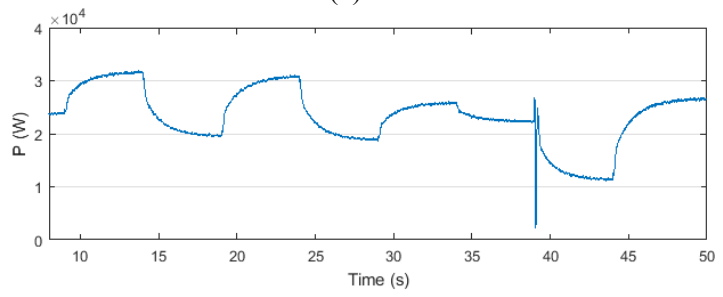

(c)

Figure 8. Load connection time span: a) frequency, b) storage active power output, c) SSMT active power output. 


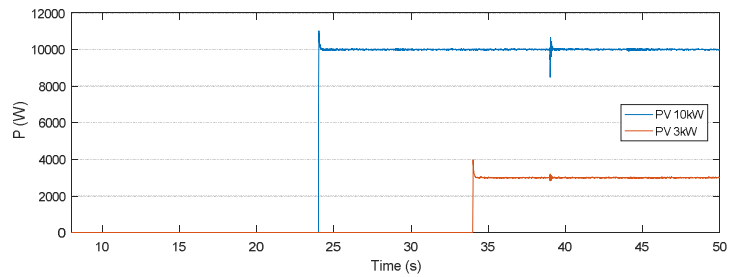

(a)

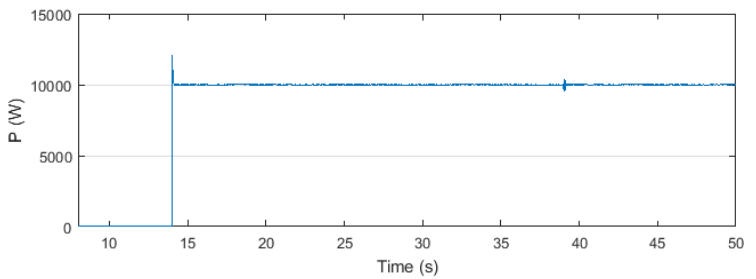

(b)

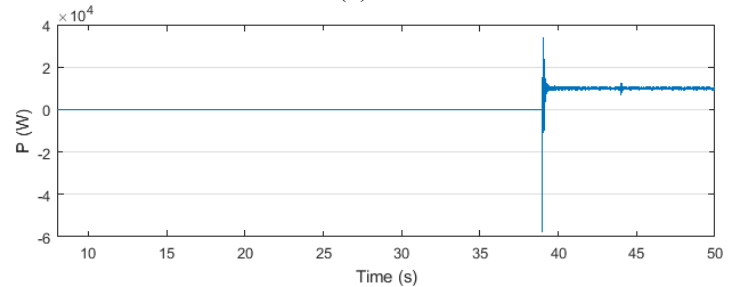

(c)

Figure 9. Load connection time span: a) PV units' active output, b) fuel cell active output, c) wind generator active output.

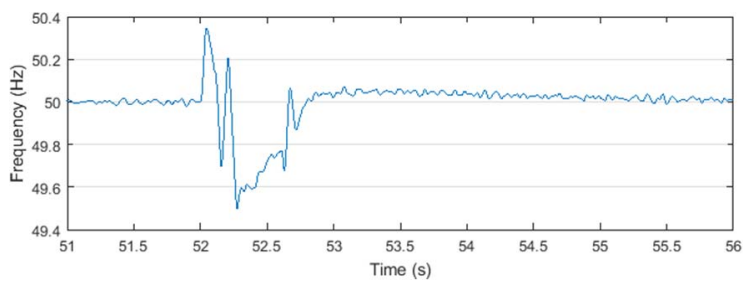

(a)

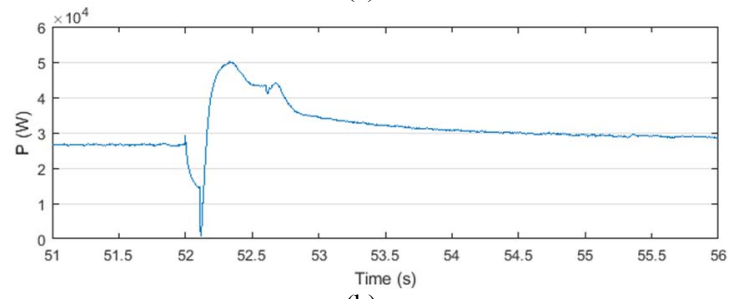

(b)

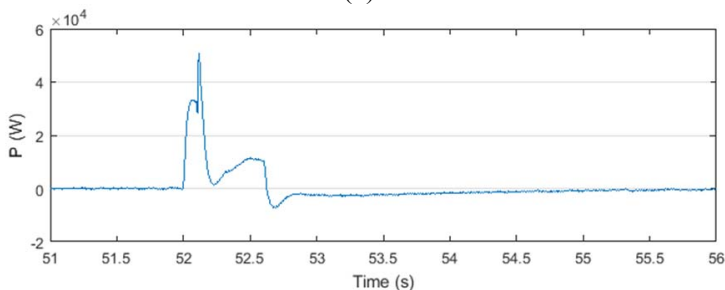

(c)

Figure 10. Short term: a) frequency, b) SSMT active power output, c) storage active power output.
This phenomenon is caused by the fact that the wind generator is modeled as an induction generator that absorbs high currents throughout its start-up procedure. The load connection sequence ends at 50 seconds and so at this stage the MG will be using the maximum capacity of every MS to supply the maximum of the load. The next stage would be connecting the MG to the medium voltage (MV) grid as soon as the latter is available in order to supply the remaining loads. However, this procedure is not simulated in this work.

\subsection{Partial Blackout}

For the partial blackout simulations, it is assumed that the MG is restored and is working in island mode, when a fault occurs at 52 seconds in branch 8 at the wind turbine terminals.

Therefore, the circuit breaker of this branch will act (at 52.1 seconds) and this part of the MG will be in partial blackout status. In branch 8 , the production of the PV and wind generator is equal to $20 \mathrm{~kW}$ and the loads in the same branch are equal to $8.02 \mathrm{~kW}$, prior to the fault. This means that $11.98 \mathrm{~kW}$ need to be shed so that the frequency can return to its nominal value.

The knapsack problem considered for the load disconnection was the same used for the load connection situation in the previous section. The only change that was necessary to do, since the knapsack is a maximization problem, was to change the priorities of the loads. For example, in the previous section was assumed that priority 3 is higher than priority 1 , but in this case priority 1 is higher than priority 3 , so the same maximization problem can be used.

The restriction of the problem (sum of the loads is less or equal to its capacity) is also valid to apply here since the objective is to shed the least amount of load possible. So, the agents will solve the knapsack problem and then, if the SSMT capacity is still exceeded, the agents solve the knapsack problem again. The load disconnection results can be found in Figure 10. After stabilizing the MG's frequency at its nominal value, then the next step is to reconnect the PV unit (at 65 seconds), wind generator (at 75 seconds) and loads of branch 8 , so the same procedure is used. The results are shown in Figure 11.

\section{Conclusions}

A decentralized MAS for tackling the MG restoration problem was proposed in this paper. Every agent was assigned to a determined load or MS, which allows the control of these elements by connecting/disconnecting them to the MG. 
The dynamic modeling of a benchmark low voltage MG was described, and then dynamic simulations were performed to assess the feasibility of the proposed MAS. In this scheme, the agents were supposed to solve a $0 / 1$ knapsack problem so that a common decision could be reached by the agents, considering the table-based dynamic programming algorithm to solve the knapsack problem. The online information was used during the restoration procedure to determine the most adequate sequence of actions. As a result, it was possible to check that the proposed scheme was feasible in both case studies.

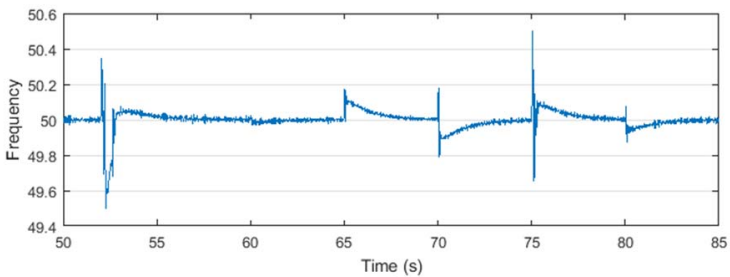

(a)

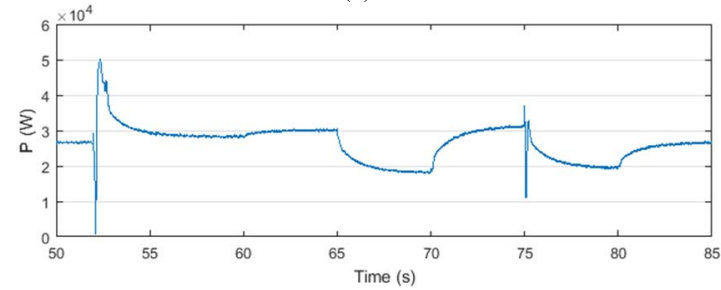

(b)

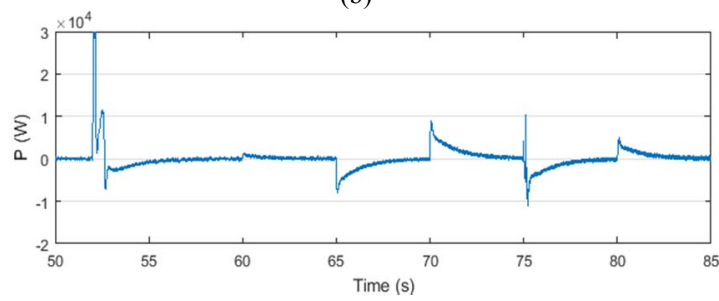

(c)

Figure 11. Long term: a) frequency, b) SSMT active power output, c) storage active power output.

\section{Acknowledgment}

J.P.S. Catalão acknowledges the support by FEDER funds through COMPETE 2020 and by Portuguese funds through FCT, under Projects SAICTPAC/0004/2015 - POCI-01-0145-FEDER-016434, and 02/SAICT/2017 - POCI-01-0145-FEDER-029803.

\section{References}

[1] Y. Liu, R. Fan, and V. Terzija, 'Power system restoration: a literature review from 2006 to 2016', J. of Modern Power Systems and Clean Energy, vol. 4, pp. 332-341, Jul. 2016.

[2] J. A. P. Lopes, C. L. Moreira, and A. G. Madureira, 'Defining control strategies for microgrids islanded operation', IEEE Transactions on Power Systems, vol. 21, no. 2, pp. 916-924, May 2006.
[3] C. L. Moreira, F. O. Resende, and J. A. P. Lopes, 'Using low voltage microgrids for service restoration', IEEE Transactions on Power Systems, vol. 22, no. 1, pp. 395-403, Feb. 2007.

[4] R. Belkacemi and A. Bababola, 'Experimental implementation of multi-agent system algorithm for distributed restoration of a smart grid system', in IEEE SOUTHEASTCON 2014, 2014, pp. 1-4.

[5] T. Nagata and K. Okamoto, 'A decentralized distribution power system restoration by using multi-agent approach', in iEECON, 2014, pp. 1-4.

[6] B. Zhao, X. Dong, and J. Bornemann, 'Service restoration for a renewable-powered microgrid in unscheduled island mode', IEEE Transactions on Smart Grid, vol. 6, no. 3, pp. 1128-1136, May 2015.

[7] A. Arab, A. Khodaei, S. K. Khator, K. Ding, V. A. Emesih, and Z. Han, 'Stochastic pre-hurricane restoration planning for electric power systems infrastructure', IEEE Transactions on Smart Grid, vol. 6, no. 2, pp. 1046-1054, Mar. 2015.

[8] A. Castillo, 'Microgrid provision of blackstart in disaster recovery for power system restoration', in 2013 SmartGridComm, pp. 534-539.

[9] J. Li, X. Y. Ma, C. C. Liu, and K. P. Schneider, 'Distribution system restoration with microgrids using spanning tree search', IEEE Transactions on Power Systems, vol. 29, no. 6, pp. 3021-3029, Nov. 2014.

[10] Z. Tan, R. Fan, Y. Liu, and L. Sun, 'Microgrid blackstart after natural disaster with load restoration using spanning tree search', in 2016 PESGM, pp. 1-5.

[11] F. O. Resende, N. J. Gil, and J. A. P. Lopes, 'Service restoration on distribution systems using multi-microgrids', Euro. Trans. Electr. Power, vol. 21, no. 2, pp. 1327-1342, Mar. 2011.

[12] Liang Che, M. Khodayar, and M. Shahidehpour, 'Only connect: microgrids for distribution system restoration', IEEE Power and Energy Magazine, vol. 12, no. 1, pp. 70-81, Jan. 2014.

[13] Y. C. Choo, K. X. Lai, M. A. Kashem, and M. Negnevitsky, "MicroGrid (MV Network) restoration using distributed resources after major emergencies," in AUPEC, vol. 1, pp. 99-104, 2005.

[14] R. Bayindir, E. Hossain, E. Kabalci, and R. Perez, 'A comprehensive study on microgrid technology', International Journal of Renewable Energy Research (IJRER), vol. 4, no. 4, pp. 1094-1107, Dec. 2014.

[15] D. E. Olivares et al., 'Trends in microgrid control', IEEE Transactions on Smart Grid, vol. 5, no. 4, pp. 19051919, Jul. 2014. 
[16] C.-S. Karavas, G. Kyriakarakos, K. G. Arvanitis, and G. Papadakis, 'A multi-agent decentralized energy management system based on distributed intelligence for the design and control of autonomous polygeneration microgrids', Energy Conversion and Management, vol. 103, pp. 166-179, Oct. 2015.

[17] C.-S. Hwang, E.-S. Kim, and Y.-S. Kim, 'A decentralized control method for distributed generations in an islanded DC microgrid considering voltage drop compensation and durable state of charge', Energies, vol. 9, no. 12, p. 1070, Dec. 2016.

[18] S. Papathanassiou, N. Hatziargyriou, K. Strunz, and others, "A benchmark low voltage microgrid network," in Proceedings of the CIGRE symposium, 2005, pp. 1-8.

[19] 'Robust maximum power point tracker using sliding mode controller for the three-phase grid-connected photovoltaic system - ScienceDirect'. [Online]. Available: https://www.sciencedirect.com/science/article/pii/S0038092 X06001113. [Accessed: 12-Feb-2018].

[20] 'Microgrid modelling and online management'. [Online]. Available: http://lib.tkk.fi/Diss/2008/isbn9789512292356/. [Accessed: 29-Apr-2018].

[21] Y. Zhu and K. Tomsovic, 'Development of models for analyzing the load-following performance of microturbines and fuel cells', Electric Power Systems Research, vol. 62, no. 1, pp. 1-11, May 2002.

[22] S. D. J. McArthur et al., 'Multi-agent systems for power engineering applications: Part I: concepts, approaches, and technical challenges', IEEE Transactions on Power Systems, vol. 22, no. 4, pp. 1743-1752, Nov. 2007.
[23] S. Jayasinghe and K. T. M. U. Hemapala, Multi agent based power distribution system restoration-a literature survey, vol. 07. 2015.

[24] G. Kyriakarakos, D. D. Piromalis, A. I. Dounis, K. G. Arvanitis, and G. Papadakis, 'Intelligent demand side energy management system for autonomous polygeneration microgrids', Applied Energy, vol. 103, pp. 39-51, Mar. 2013.

[25] E. Rokrok, M. Shafie-khah, P. Siano, and J. P. S. Catalão, 'A decentralized multi-agent-based approach for low voltage microgrid restoration', Energies, vol. 10, no. 10, p. 1491, Sep. 2017.

[26] M. Posypkin and S. T. T. Sin, 'Comparative analysis of the efficiency of various dynamic programming algorithms for the knapsack problem', in 2016 EIConRusNW, pp. 313316.

[27] S. Pushpa K, T. V. Mrunal, and C. Suhas, "A Study of Performance Analysis on Knapsack Problem", National Conference on "Recent Trends in Information Technology" (NCRTIT), 2016.

[28] K. Samarakoon, J. Ekanayake, and N. Jenkins, 'Investigation of domestic load control to provide primary frequency response using smart meters', IEEE Transactions on Smart Grid, vol. 3, no. 1, pp. 282-292, Mar. 2012.

[29] C. Gouveia, J. Moreira, C. L. Moreira, and J. A. P. Lopes, 'Coordinating storage and demand response for microgrid emergency operation', IEEE Transactions on Smart Grid, vol. 4, no. 4, pp. 1898-1908, Dec. 2013. 